\title{
Low-intensity extracorporeal shockwave therapy for erectile dysfunction: an overview of systematic reviews
}

\author{
Fan Yuan ${ }^{1,2 \#}$, Yang Wang ${ }^{3 \#}$, Ziyang Ma ${ }^{1 \#}$, Mingyi Jing ${ }^{1,2 \#}$, Yaodong You ${ }^{1,2}$, Xujun Yu ${ }^{2,4}$, Degui Chang ${ }^{1}$, \\ Peihai Zhang ${ }^{1}$ \\ ${ }^{1}$ Traditional Chinese Medicine Regulating Metabolic Diseases Key Laboratory of Sichuan Province, Hospital of Chengdu University of Traditional \\ Chinese Medicine, Chengdu, China; ${ }^{2}$ Department of Clinical Medicine, Chengdu University of Traditional Chinese Medicine, Chengdu, China; \\ ${ }^{3}$ Department of Urology, the Affiliated Hospital of Chengdu University, Chengdu, China; ${ }^{4}$ Department of Andrology, the Reproductive \& Women's \\ and Children's Hospital, Chengdu University of Traditional Chinese Medicine, Chengdu, China \\ Contributions: (I) Conception and design: F Yuan, Y Wang, P Zhang; (II) Administrative support: Y You, X Yu, D Chang, P Zhang; (III) Provision of \\ study materials or patients: Y Wang, Y You, X Yu, D Chang; (IV) Collection and assembly of data: Z Ma, M Jing, Y You, X Yu; (V) Data analysis and \\ interpretation: F Yuan, Y Wang, Z Ma, M Jing; (VI) Manuscript writing: All authors; (VII) Final approval of manuscript: All authors. \\ \#These authors contributed equally to this work and are joint first authors. \\ Correspondence to: Peihai Zhang. Traditional Chinese Medicine Regulating Metabolic Diseases Key Laboratory of Sichuan Province, Hospital of \\ Chengdu University of Traditional Chinese Medicine, Chengdu, China. Email: zhangpeihai@126.com.
}

Background: Low-intensity extracorporeal shockwave therapy (LI-ESWT) may be a successful complementary treatment approach for erectile dysfunction (ED). In this study, we aimed to review and summarize the research evidence from systematic reviews (SRs)/meta-analyses (MAs) regarding the clinical effectiveness of LI-ESWT for ED.

Methods: Studies on LI-ESWT for ED were searched using eight electronic databases from establishment of each database to 31 June 2021 with the language restrictions of Chinese and English. All articles were screened, and qualifying data were recorded based on the inclusion criteria. Methods including: the Assessing the Methodological Quality of Systematic Reviews 2 (AMSTAR-2); the Risk of Bias in Systematic Reviews (ROBIS); the Preferred Reporting Items for Systematic Reviews and Meta-Analyses (PRISMA); and Grading of Recommendations, Assessment Development, and the Evaluation (GRADE) were used by two independent raters to assess methodological quality, risk of bias, reporting quality, and SR evidence of quality, respectively.

Results: Eight SRs/MAs met all inclusion criteria. Seven reviews were rated as critically low on overall confidence and one review was low on confidence based on the AMSTAR-2 appraisal tool. While most PRISMA criteria were met, the major reporting flaws were in relation the financial statements not being included, along with no protocol registrations. Three SRs/MAs were classed as low risk regarding bias as measured by the ROBIS tool. Based on the GRADE method, only one SRs/MAs of high-quality evidence and seven SRs/MAs of moderate-quality evidence were found. The present research results supported LIESWT as a complementary therapy for ED patients, but the evidence should be considered carefully due to the methodological flaws identified.

Discussion: Our results showed that LI-ESWT as an adjunctive therapy has benefits for ED patients. There were no obvious side effects, and the number of shockwave treatments and energy flux density (EFD) would affect the IIEF-EF, EHS and PSV scores. However, due to the limited sample size and the quality of reporting evidence, our conclusions may not be fully representative.

Keywords: Erectile dysfunction (ED); low-intensity extracorporeal shockwave therapy; overview; systematic reviews (SRs)

Submitted Jul 30, 2021. Accepted for publication Sep 16, 2021.

doi: 10.21037/tau-21-730

View this article at: https://dx.doi.org/10.21037/tau-21-730 


\section{Introduction}

Erectile dysfunction (ED), common in adult men older than 40 years, indicates the incapacity to attain or sustain penile erection for a sufficient period to achieve successful sexual intercourse $(1,2)$. The prevalence of ED increases with age and comorbid conditions. ED not only seriously affects the quality of patients' lives, but may also be an early symptom of cardiovascular disease $(3,4)$. Clinical diagnosis of ED is usually made using validated questionnaires. The Erectile Dysfunction Inventory of Treatment Satisfaction (EDITS) and International Index of Erectile Function (IIEF) are the most frequently used questionaries. In addition to assessing the nature of $\mathrm{ED}$ in individual patients, these questionnaires can also be used for monitoring improvements of symptoms and outcomes of treatments. Besides, the objective methods to make a diagnosis for ED include nocturnal penile tumescence and rigidity (NPTR), audiovisual sexual stimulation (AVSS), penile Intracavernosal injection (ICI), Color Doppler duplex ultrasound (CDDU), Penile Dynamic Infusion Cavernosometry and Cavernosography, etc. Meanwhile, considering the common risk factors between ED and some cardiovascular diseases, laboratory tests, such as blood routine and blood biochemistry index, are also essential. Most of the meta-analyses included in this paper took IIEF and EHS questionnaire survey as outcome indicators, and did not make statistical analysis on other objective indicators.

Penile erection is a complex physiological phenomenon that is precisely regulated and coordinated by nerve, endocrine, blood vessel and penile cavernous tissue, including the filling of penile artery, relaxation of trabecular smooth muscle, cavernous venous occlusion, etc. (1). Mental factors also play an important role in erectile function. Any problem in the above process may cause ED. Epidemiological data showed that the prevalence of ED increased with age $(5,6)$. Obesity, diabetes, smoking and lack of exercise are also risk factors $(4,7)$. In addition, premature ejaculation, low urinary tract symptoms (LUTS) and benign prostatic hyperplasia (BPH) are related to sexual dysfunction (8).

The treatment principle of ED is to obtain a satisfactory sexual life through individualized comprehensive treatment. The first-line drugs for ED are phosphodiesterase type 5 (PDE5) inhibitors (2), and although they are effective, the relief is only temporary, and they provide no permanent improvement. In addition, the side-effects caused by PDE5 inhibitors, such as visual impairment, dyspepsia, myalgia, and back pain, are difficult for patients to tolerate. Especially for patients taking antihypertensive drugs, the combination of PDE5 inhibitors will have a slight synergistic effect (9). Some patients choose other treatments due to PDE5 inhibitors not working for them, such as penile prosthesis implantation, intracorporal injection, etc. (2). Such treatment modalities are used as second- or even third-line approaches, yet long-term use is associated with complications and unwanted side-effects (10). Therefore, they are not the best choice for treating ED.

Extracorporeal shock wave (ESW) is a special sound wave carrying energy, which has little damage to human connective tissue, skin, fat, muscle and other tissues. ESW is divided into high, medium and low strength grades (11). According to the energy density of the second focus of ESW: higher than $0.60 \mathrm{~mJ} / \mathrm{mm}^{2}$ is high energy; $0.28-0.60 \mathrm{~mJ} / \mathrm{mm}^{2}$ is medium energy; $0.08-0.28 \mathrm{~mJ} / \mathrm{mm}^{2}$ is low energy. The functions and application scope of ESW with different strength are different. Because high-strength ESW has focused mechanical damage characteristics, it should be used in lithotripsy treatment. Medium energy ESW has anti-inflammatory function, so it is mostly used in surgery, such as tendinitis, fracture nonunion, synovial bursitis, etc. Low energy ESW can promote angiogenesis. Its focusing effect can cause minor damage and mechanical pressure, produce biological effects, release angiogenesis factors, promote the angiogenesis of target tissue, and then improve its blood supply. It is mainly used in cardiovascular disease, chronic trauma and peripheral neuropathy (12).

In recent years, LI-ESWT has been considered to be a non-invasive, effective treatment for ED. Shockwaves can penetrate tissues and target precise areas or organs to achieve clinically significant changes (13). Vardi et al. first report the application of LI-ESWT in treating ED in 2010 (13). After being subjected to clinical observation for eight years, LI-ESWT has gradually become an effective treatment for ED. Furthermore, there is evidence that LI-ESWT is tolerable and effective for patients with ED and can significantly improve blood flow of the penis, thus improving mild ED $(14,15)$. The mechanisms of LI-ESWT include: (I) LI-ESWT can effectively induce angiogenesis, increase the expression of VEGF and other angiogenic factors, promote the formation of blood vessels in the corpus cavernosum of the penis, and cause penis hyperemia, thus promoting penis erection (16); and (II) LIESWT is conducive to remodeling of cavernous tissue in smooth muscle cells, increases penile microvascularization (17), induces muscle cell differentiation (18), and improves 
erection. Hence, some patients choose to undergo LIESWT for treating ED to improve their quality of life, but evidence supporting the benefits and safety of LI-ESWT remains absent. Therefore, high-quality and credible clinical evidence on LI-ESWT for treating ED is still required. The novelty of this article is that the research on the treatment of ED with LI-ESWT has a wider range of $\mathrm{ED}$, reviews all published meta-analyses, includes the latest literatures and has a higher quality of evidence.

Systematic reviews (SRs) and meta-analyses (MAs) are the highest level of evidence in the evidence-based medicine pyramid to help researchers identify, evaluate, and compile existing research-based data and literature (19). In past years, although the number of SRs/MAs is increasing, inconsistencies across SRs/MAs exist due to the varying quality of primary studies included or methodological flaws. Consequently, there is skepticism around the credibility of such evidence. Systematic overviews of SRs/MAs are a relatively novel tool for combining the results of multiple SRs/MAs, assessing their quality, and addressing any inconsistencies. Given these characteristics of systematic overviews of SRs/MAs, we conducted an overview to analytically evaluate the research quality of relevant SRs/ MAs concerning the clinical usage of LI-ESWT in treating ED. We present the following article in accordance with the PRISMA reporting checklist (available at https://dx.doi. org/10.21037/tau-21-730).

\section{Methods}

\section{Inclusion criteria}

\section{Types of studies}

Our systematic overview included SRs/MAs of randomized controlled trials (RCTs), and patients must have been diagnosed with ED based on definitive diagnostic criteria. Duplicate papers, university dissertations, and those SRs/ MAs that were not academically credible were not included.

\section{Type of participants}

Participants were diagnosed as having ED according to any international or national diagnostic criteria. There were no limitations on ethnicity, age, gender, and the duration and stage of the disease.

\section{Type of interventions}

Studies of LI-ESWT or LI-ESWT plus PDE5 inhibitor (PDE5i) as an intervention for ED were included. The control group included the following treatments: sham therapy with shock wave probes, other treatments, or no treatment. SRs of LI-ESWT joined with other treatments (such as vacuum erection device, VED) were excluded.

\section{Evaluation of treatment outcomes}

Because the literatures included in this paper took the changes of IIEF and EHS scores before and after treatment, peak systolic velocity (PSV) and adverse reactions as outcome indicators, this article was also based on this standard. The primary treatment outcomes were evaluated using the International Index of Erectile Function Erectile Function Domain score (IIEF-EF) and the Erection Hardness Score (EHS). The secondary outcomes were peak systolic velocity (PSV) and adverse reactions.

\section{Data sources and search strategy}

The Chinese and English databases were searched from inception to 31 June 2021, including Embase, PubMed, Cochrane Library, Web of Science, VIP Journals Database, Chinese Biomedical Databases (CBM), China National Knowledge Infrastructure (CNKI), and Wanfang Databases. The search terms used were as follows: "shockwave OR shock wave" AND "systematic review OR meta-analysis" AND "erectile dysfunction OR ED" (erectile dysfunction as a mesh term). As for searching in Chinese databases, the same search terms were used in Chinese.

\section{Data handling and extraction}

All papers were examined by two independent raters. According to the predefined criteria, two raters also validated and extracted data from papers. During the process of data extraction, disagreements were resolved through discussion with a third investigator.

\section{Quality assessment}

Two independent authors used the Assessing the Methodological Quality of Systematic Reviews 2 (AMSTAR-2) tool to evaluate the methodological quality of the SRs included (20), the Preferred Reporting Items for Systematic Reviews and Meta-Analyses (PRISMA) tool to examine the reporting quality $(21,22)$, the Risk of Bias in Systematic Reviews (ROBIS) tool to assess the risk of bias (23), and the Grading of Recommendations, Assessment Development, and the Evaluation (GRADE) 

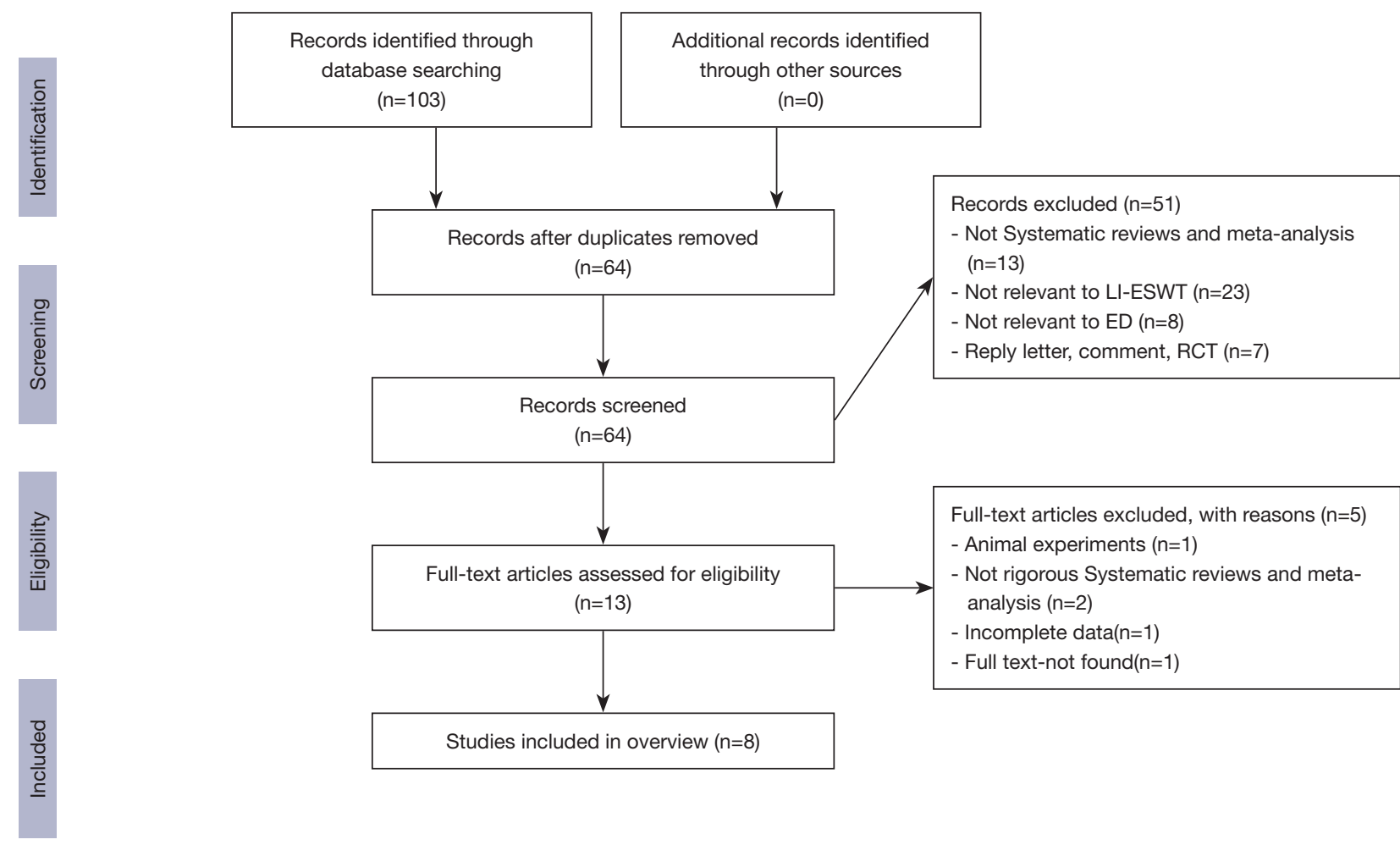

Figure 1 PRISMA flow chart of the study selection process.

tool for evaluating the quality of evidence (24). Disagreements among authors were resolved through discussion with a third investigator.

The AMSTAR-2 tool has 16 items, and each item was answered with a "yes" (a positive result), a "partial yes" (partial adherence to the standard) or a "no" (no information provided to rate an item) based on the level of adherence to the standard (20). After assessing the flaws found in all items, the general quality of the work can be classed as "high," "moderate," "low," or "critically low". The PRISMA statement has 27 items covering seven aspects of SRs, which include titles, abstracts, introductions, methods, results, discussions, and funding $(21,22)$, and each item was answered with "yes" (representing full reports), "partial yes" (representing partially compliant reports) and "no" (representing no report). The ROBIS tool consists of three phases for assessing the level of bias in an SR, which are classed as "low," "high," or "unclear" (23). The GRADE system is graded into four levels: "high", "moderate", "low" or "very low". The initial grading is based on five aspects: study limitations, indirectness of evidence, inconsistency of results, lack of precision, and publication bias $(25,26)$.

\section{Statistical analysis}

This paper summarizes the data of the included systematic review (SRs)/meta-analysis (MAs), so a descriptive analysis is carried out.

\section{Results}

\section{Overview of the screening process}

In total, 103 studies were selected from the eight databases. After duplicates were removed, 39 studies remained. Fiftyone studies were excluded after title/abstract screening. Eventually, eight selected studies were included in the present overview. The entire screening and selection process is depicted in Figure 1.

\section{Description of characteristics}

In total, eight eligible SRs with 62 randomized controlled trials (RCTs) were included in the present study, and the data extracted are shown in Table 1. These SRs/MAs included in the study were published between 2017 and 
Table 1 Main characteristics of the included reviews

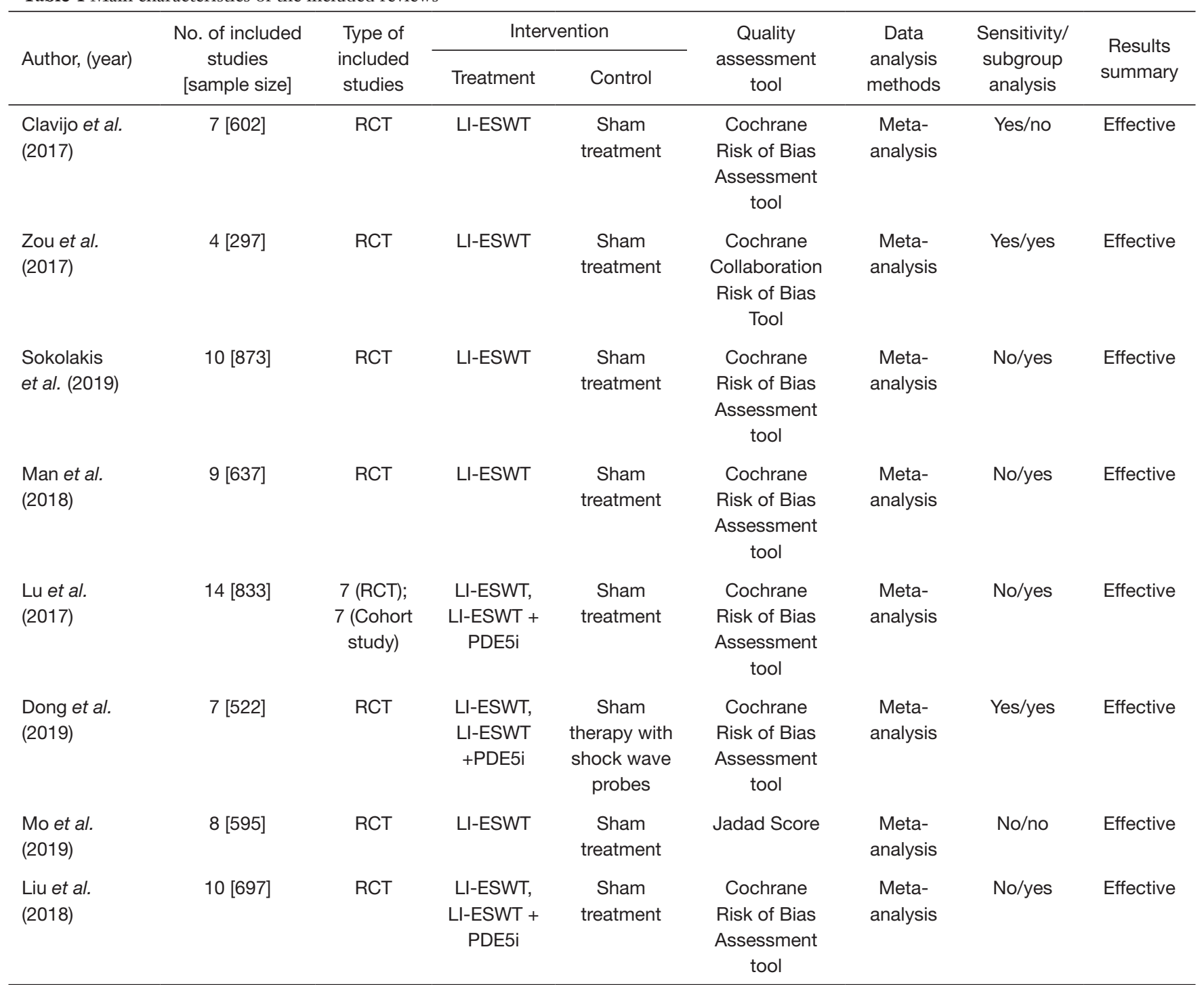

RCT, randomized controlled trial; LI-ESWT, low-intensity extracorporeal shockwave therapy; PDE5i, phosphodiesterase type 5 inhibitor.

2021, of which six were written in English (14,27-31) and two in Chinese (32,33). All included SRs evaluated the efficacy of LI-ESWT for ED. In the treatment group LIESWT was used, whereas shame therapy using shock wave probes was mainly used in the control group. The number of RCTs included in each SR ranged between 4 and 14, and the sample size ranged from 297 to 873 patients. In terms of quality assessment scales, only one (32) used the Jadad score, and the Cochrane Collaboration Risk of Bias Tool was used in all others. Three SRs/MAs conducted a sensitivity analysis $(14,27,31)$. Subgroup analysis was performed in six SRs/MAs $(27-31,33)$. All SRs/MAs showed the efficiency of LI-ESWT in treating ED.

\section{Results on methodological quality}

Table 2 displays a summary of the methodological quality using the AMSTAR-2 instrument, which is developed from AMSTAR and contains 16 items. Items 2, 4, 7, 9, 11, 13, and 15 are used to critically evaluate the effectiveness of an SR. Considering that several critical items of the included SRs/ MAs were not met, the final evaluation of methodological quality was critically low for seven articles and low for one article. The limitations of methodology come from the 
Table 2 Results of the AMSTAR-2 assessments (20)

Item
1. Did the research questions and inclusion criteria for the
review include the components of PICO?
2. Did the report of the review contain an explicit statement
that the review methods were established prior to the
conduct of the review and did the report justify any
significant deviations from the protocol?

3. Did the review authors explain their selection of the study designs for inclusion in the review?

4. Did the review authors use a comprehensive literature search strategy?

5. Did the review authors perform study selection in duplicate?

6. Did the review authors perform data extraction in duplicate?

7. Did the review authors provide a list of excluded studies and justify the exclusions?

8. Did the review authors describe the included studies in adequate detail?

9. Did the review authors use a satisfactory technique for assessing the risk of bias $(\mathrm{RoB})$ in individual studies that were included in the review?

10. Did the review authors report on the sources of funding for the studies included in the review?

11. If meta-analysis was performed, did the review authors use appropriate methods for statistical combination of results?

12. If meta-analysis was performed, did the review authors assess the potential impact of RoB in individual studies on the results of the meta-analysis or other evidence synthesis?

13. Did the review authors account for RoB in primary studies when interpreting/discussing the results of the review?

14. Did the review authors provide a satisfactory explanation for, and discussion of, any heterogeneity observed in the results of the review?

15. If they performed quantitative synthesis, did the review authors carry out an adequate investigation of publication bias (small study bias) and discuss its likely impact on the results of the review?

16. Did the review authors report any potential sources of conflict of interest, including any funding they received for conducting the review?

Quality

Clavijo
et al.
$(2017)$

$\begin{array}{cc}\text { Zou } & \text { Sokola } \\ \text { et al. } & \text { et al. } \\ (2017) & (2019)\end{array}$

$\begin{array}{ccccc}\text { Man } & \text { Lu } & \text { Dong } & \text { Mo } & \text { Liu } \\ \text { et al. } & \text { et al. } & \text { et al. } & \text { et al. } & \text { et al. } \\ (2018) & (2017) & (2019) & (2019) & (2018)\end{array}$

Y

$Y \quad Y$

Y

$\mathrm{N}$

N

N

N

N

N

N

N

\begin{tabular}{|c|c|c|c|c|c|c|c|}
\hline Y & $Y$ & $\mathrm{Y}$ & $Y$ & $Y$ & $Y$ & $Y$ & $Y$ \\
\hline Y & PY & $Y$ & PY & PY & $Y$ & PY & PY \\
\hline Y & $Y$ & $Y$ & $Y$ & $Y$ & $\mathrm{Y}$ & $Y$ & $\mathrm{Y}$ \\
\hline Y & $Y$ & $\mathrm{Y}$ & $Y$ & $Y$ & $\mathrm{Y}$ & PY & $\mathrm{Y}$ \\
\hline$N$ & $\mathrm{~N}$ & PY & $\mathrm{N}$ & $\mathrm{N}$ & $\mathrm{Y}$ & $\mathrm{N}$ & $\mathrm{N}$ \\
\hline Y & $\mathrm{Y}$ & $Y$ & $Y$ & $Y$ & $\mathrm{Y}$ & $Y$ & $\mathrm{Y}$ \\
\hline Y & $Y$ & $Y$ & $Y$ & $\mathrm{Y}$ & $\mathrm{Y}$ & $Y$ & $\mathrm{Y}$ \\
\hline Y & $Y$ & $\mathrm{~N}$ & $Y$ & $Y$ & $\mathrm{Y}$ & $\mathrm{N}$ & $\mathrm{N}$ \\
\hline Y & $Y$ & $Y$ & $Y$ & $Y$ & $\mathrm{Y}$ & $Y$ & $\mathrm{Y}$ \\
\hline$N$ & $Y$ & $Y$ & $\mathrm{Y}$ & $\mathrm{Y}$ & $\mathrm{Y}$ & PY & $\mathrm{Y}$ \\
\hline Y & $Y$ & $Y$ & $\mathrm{~N}$ & $Y$ & $\mathrm{Y}$ & $\mathrm{N}$ & $\mathrm{N}$ \\
\hline Y & PY & $Y$ & $Y$ & $Y$ & $Y$ & $Y$ & $\mathrm{Y}$ \\
\hline Y & $\mathrm{Y}$ & $\mathrm{N}$ & $\mathrm{N}$ & $\mathrm{Y}$ & $\mathrm{Y}$ & $\mathrm{Y}$ & $\mathrm{N}$ \\
\hline $\mathrm{N}$ & $Y$ & $Y$ & $Y$ & $Y$ & $Y$ & $\mathrm{~N}$ & $\mathrm{~N}$ \\
\hline
\end{tabular}

PICO, Patients, Intervention, Comparison, Outcomes; Y, yes; PY, partial yes; N, no; CL, critically low; L, low; H, high. https://amstar.ca/ Amstar_Checklist.php 
Table 3 Results of the PRISMA

\begin{tabular}{|c|c|c|c|c|c|c|c|c|c|}
\hline Section/Topic & Items & $\begin{array}{c}\text { Clavijo } \\
\text { et al. } \\
(2017)\end{array}$ & $\begin{array}{l}\text { Zou } \\
\text { et al. } \\
(2017)\end{array}$ & $\begin{array}{c}\text { Sokola } \\
\text { et al. } \\
(2019)\end{array}$ & $\begin{array}{l}\text { Man } \\
\text { et al. } \\
(2018)\end{array}$ & $\begin{array}{l}\text { Lu } \\
\text { et al. } \\
\text { (2017) }\end{array}$ & $\begin{array}{l}\text { Dong } \\
\text { et al. } \\
(2019)\end{array}$ & $\begin{array}{l}\text { Mo } \\
\text { et al. } \\
\text { (2019) }\end{array}$ & $\begin{array}{l}\text { Liu } \\
\text { et al. } \\
\text { (2018) }\end{array}$ \\
\hline Abstract & 2. Structured summary & $\mathrm{Y}$ & $\mathrm{Y}$ & $\mathrm{Y}$ & $\mathrm{Y}$ & $\mathrm{Y}$ & $\mathrm{Y}$ & $\mathrm{Y}$ & $\mathrm{Y}$ \\
\hline Introduction & 3. Rationale & $\mathrm{Y}$ & $\mathrm{Y}$ & $\mathrm{Y}$ & $\mathrm{Y}$ & Y & Y & $\mathrm{Y}$ & $\mathrm{Y}$ \\
\hline \multirow[t]{10}{*}{ Methods } & 5. Protocol and registration & $\mathrm{N}$ & $\mathrm{N}$ & $\mathrm{N}$ & $\mathrm{N}$ & $\mathrm{N}$ & $\mathrm{N}$ & $\mathrm{N}$ & $\mathrm{N}$ \\
\hline & 6. Eligibility criteria & $\mathrm{Y}$ & $\mathrm{Y}$ & Y & Y & Y & Y & Y & $\mathrm{Y}$ \\
\hline & 7. Information sources & Y & PY & Y & Y & PY & Y & $\mathrm{Y}$ & $\mathrm{Y}$ \\
\hline & 8. Search & PY & $\mathrm{N}$ & PY & Y & Y & Y & PY & PY \\
\hline & 11. Data items & Y & Y & Y & Y & Y & Y & Y & Y \\
\hline & 12. Risk of bias in individual studies & $\mathrm{Y}$ & $\mathrm{Y}$ & Y & Y & Y & Y & $\mathrm{Y}$ & $\mathrm{Y}$ \\
\hline & 13. Summary measures & Y & Y & Y & Y & $\mathrm{Y}$ & $\mathrm{Y}$ & $\mathrm{Y}$ & Y \\
\hline & 14. Synthesis of results & $\mathrm{Y}$ & $\mathrm{Y}$ & $\mathrm{Y}$ & $\mathrm{Y}$ & $\mathrm{Y}$ & $\mathrm{Y}$ & $\mathrm{Y}$ & $\mathrm{Y}$ \\
\hline & 15. Risk of bias across studies & PY & $\mathrm{N}$ & $\mathrm{Y}$ & $\mathrm{Y}$ & Y & $\mathrm{Y}$ & $\mathrm{N}$ & $\mathrm{N}$ \\
\hline & 16. Additional analyses & $\mathrm{N}$ & PY & Y & PY & Y & PY & PY & PY \\
\hline \multirow[t]{2}{*}{ Results } & 17. Study selection & $\mathrm{Y}$ & Y & $\mathrm{Y}$ & Y & $\mathrm{Y}$ & Y & Y & Y \\
\hline & 23. Additional analysis & $\mathrm{N}$ & Y & PY & PY & $\mathrm{N}$ & $\mathrm{N}$ & $\mathrm{N}$ & $\mathrm{Y}$ \\
\hline \multirow[t]{3}{*}{ DISCUSSION } & 24. Summary of evidence & Y & Y & Y & Y & Y & Y & Y & $\mathrm{Y}$ \\
\hline & 25. Limitations & $\mathrm{Y}$ & Y & $\mathrm{Y}$ & PY & $\mathrm{N}$ & $\mathrm{Y}$ & PY & $\mathrm{Y}$ \\
\hline & 26. Conclusions & $\mathrm{Y}$ & $\mathrm{Y}$ & Y & Y & Y & Y & $\mathrm{Y}$ & $\mathrm{Y}$ \\
\hline Funding & 27. Funding & $\mathrm{N}$ & $\mathrm{Y}$ & $\mathrm{N}$ & Y & Y & Y & $\mathrm{N}$ & $\mathrm{N}$ \\
\hline
\end{tabular}

Y, yes; PY, partial yes; N, no; CL, critically low; L, low; H, high.

following items: item 2 (no SR/MA registered the protocol), item 4 (only three SRs/MAs provided a complete search strategy), item 13 [three SRs/MAs did not take the risk of bias $(\mathrm{RoB})$ into account in the interpretation of the results from primary studies], and item 15 (three SRs/MAs did not appraise publication bias or debate its respective influence on the review).

\section{Results on reporting quality}

An outline of reporting quality using the PRISMA tool is illustrated in Table 3. The PRISMA checklist includes seven sections: titles, abstracts, introductions, methods, results, discussion, and funding (21). Generally, the reporting was relatively comprehensive, but there were 
Table 4 Results of the ROBIS assessments

\begin{tabular}{|c|c|c|c|c|c|c|}
\hline \multirow[b]{2}{*}{ Review } & \multirow{2}{*}{$\begin{array}{c}\text { Phase } 1 \\
\text { Assessing relevance } \\
\text { (participants, interventions, } \\
\text { comparisons, outcomes) }\end{array}$} & \multicolumn{4}{|c|}{ Phase 2} & \multirow{2}{*}{$\begin{array}{c}\text { Phase } 3 \\
\text { Risk } \\
\text { of bias } \\
\text { in the } \\
\text { review }\end{array}$} \\
\hline & & $\begin{array}{c}\text { Domain } 1 . \\
\text { Study eligibility } \\
\text { criteria }\end{array}$ & $\begin{array}{l}\text { Domain } 2 . \\
\text { Identification } \\
\text { and selection of } \\
\text { studies }\end{array}$ & $\begin{array}{l}\text { Domain3. } \\
\text { Data collection } \\
\text { and study } \\
\text { appraisal }\end{array}$ & $\begin{array}{l}\text { Domain } 4 . \\
\text { Synthesis and } \\
\text { findings }\end{array}$ & \\
\hline Clavijo et al. (2017) & (:) & (:) & (:) & (:) & : : & (:) \\
\hline Zou et al. (2017) & (:) & : : & (:) & (:) & ;) & : : \\
\hline Sokolakis et al. (2019) & (:) & (:) & ;) & (:) & : : & ;) \\
\hline Man et al. (2018) & (:) & ;) & : & (:) & $?$ & (:) \\
\hline Lu et al. (2017) & (:) & ;) & $?$ & ;) & 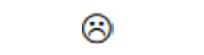 & 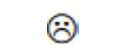 \\
\hline Dong et al. (2019) & (:) & ;) & : & ;) & ;) & 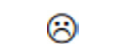 \\
\hline Mo et al. (2019) & (:) & ;) & ;) & 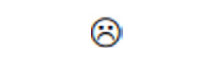 & 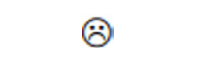 & 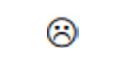 \\
\hline Liu et al. (2018) & (:) & ;) & $?$ & : & (:) & 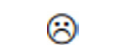 \\
\hline
\end{tabular}

(:), low risk; $:$, high risk; ?, unclear risk.

still some defects. In the section on methods, no SRs/ MAs reported the topic of the protocol and registration. Three SRs/MAs (29-31) presented detailed search information, risk of bias across studies was reported in four SRs/MAs (28-31), and only two SRs/MAs $(28,30)$ presented additional analyses. In the discussion part of the studies, limitations were presented in five SRs/MAs $(14,27,28,31,33)$. Funding was reported in four SRs/MAs $(27,29-31)$.

\section{Results on ROBIS evaluation}

Regarding ROBIS, all SRs/MAs were evaluated to have a low risk of bias in Phase 1, which evaluated relevance of the research topic. Phase 2 had four domains. For assessing the eligibility criteria of studies in Domain 1, seven SRs/MAs $(14,28-33)$ showed a low risk of bias. Domain 2 investigated the identification and inclusion of studies, and four SRs/ MAs $(14,27,28,32)$ were at a low risk of bias. Assessing the data collection and study appraisal constituted Domain 3, in which six SRs/MAs $(14,27-31)$ were classed as low risk of bias. For Domain 4, which evaluated synthesis and findings, four SRs/MAs $(14,28,30,32)$ were at high risk of bias. In Phase 3, the overall risk of bias was considered, and three SRs/MAs $(14,28,29)$ had a low risk. Table 4 presents more detailed information.

\section{Results on evidence quality}

This review includes eight SRs/MAs involving 20 outcomes. One outcome was high-quality evidence, seven outcomes moderate-quality evidence, and eight low-quality evidence. Due to the elevated risk of bias, lack of precision, inconsistent results, and indirectness, the evidence was downgraded. More details are provided in Table 5.

\section{Efficacy evaluation}

Eight SRs/MAs compared the changes of IIEF-EF scores of LI-ESWT with those of sham treatment. The efficacy of LI-ESWT yielded superior results compared with sham treatment in the treatment of ED. Three SRs/MAs $(29,30,33)$ showed that the clinical effectiveness of LIESWT in treating ED was better when other diseases were absent. Two SRs/MAs $(27,28)$ showed that LI-ESWT for ED patients with ineffective PDE5i was more effective than for ED patients with effective PDE5i. Three SRs/Mas $(29,30,33)$ showed that the clinical efficacy for ED of LIESWT of shorter duration (4-6 weeks) was better than that of longer duration (9 weeks). However, one SRs/MAs (32) 
Table 5 Results of evidence quality

\begin{tabular}{|c|c|c|c|c|c|c|c|c|c|}
\hline References & Outcomes & \multicolumn{5}{|c|}{ Certainty assessment } & $\begin{array}{l}\text { Relative effect } \\
\qquad(95 \% \mathrm{Cl})\end{array}$ & $P$ value & Certainty \\
\hline \multirow[t]{2}{*}{$\begin{array}{l}\text { Zou et al. } \\
(2017)\end{array}$} & IIEF-EF & Not serious & Serious & Not serious & Serious & None & $\begin{array}{c}\text { RR } 2.50 \\
(0.74-8.54)\end{array}$ & 0.02 & $\begin{array}{l}\text { Low: } \\
\oplus \oplus \bigcirc \bigcirc\end{array}$ \\
\hline & $\begin{array}{l}\text { Adverse } \\
\text { effects }\end{array}$ & \multicolumn{8}{|c|}{ No statistical analysis was performed } \\
\hline \multirow[t]{3}{*}{$\begin{array}{l}\text { Sokolakis } \\
\text { et al. (2019) }\end{array}$} & IIEF-EF & Not serious & Serious & Not serious & Not serious & None & $\begin{array}{c}\text { MD } 3.97 \\
(2.09-5.84)\end{array}$ & $<0.0001$ & $\begin{array}{c}\text { Moderate: } \\
\oplus \oplus \oplus \bigcirc\end{array}$ \\
\hline & PSV & serious & Not serious & Not serious & Serious & None & $\begin{array}{c}\text { MD } 4.12 \\
(2.30-5.94)\end{array}$ & $\leq 0.00001$ & $\begin{array}{l}\text { Low: } \\
\oplus \oplus \bigcirc \bigcirc\end{array}$ \\
\hline & $\begin{array}{l}\text { Adverse } \\
\text { effects }\end{array}$ & \multicolumn{8}{|c|}{ No statistical analysis was performed } \\
\hline \multirow[t]{2}{*}{$\begin{array}{l}\text { Man et al. } \\
(2018)\end{array}$} & IIEF & Serious & Serious & Not serious & Not serious & None & $\begin{array}{c}\text { MD } 2.54 \\
(0.83-4.25)\end{array}$ & 0.004 & $\begin{array}{l}\text { Low: } \\
\oplus \oplus \bigcirc \bigcirc\end{array}$ \\
\hline & EHS & Serious & Serious & Not serious & Not serious & None & $\begin{array}{c}\text { RD } 0.38 \\
(0.07-0.69)\end{array}$ & 0.02 & $\begin{array}{l}\text { Low: } \\
\oplus \oplus \bigcirc \bigcirc\end{array}$ \\
\hline $\begin{array}{l}\text { Lu et al. } \\
\text { (2017) }\end{array}$ & IIEF & Not serious & Not serious & Not serious & Serious & None & $\begin{array}{c}\text { MD } 2.00 \\
(0.99-3.00)\end{array}$ & $<0.0001$ & $\begin{array}{c}\text { Moderate: } \\
\oplus \oplus \oplus \bigcirc\end{array}$ \\
\hline $\begin{array}{l}\text { Dong et al. } \\
\text { (2019) }\end{array}$ & $\begin{array}{l}\text { Adverse } \\
\text { effects }\end{array}$ & \multicolumn{8}{|c|}{ No statistical analysis was performed } \\
\hline \multirow[t]{2}{*}{$\begin{array}{l}\text { Mo et al. } \\
(2019)\end{array}$} & IIEF & Serious & Not serious & Not serious & Serious & None & $\begin{array}{l}\text { WMD } 1.70 \\
(0.44,2.96)\end{array}$ & 0.008 & $\begin{array}{l}\text { Low: } \\
\oplus \oplus \bigcirc \bigcirc\end{array}$ \\
\hline & EHS & Serious & Not serious & Not serious & Not serious & None & $\begin{array}{c}\text { RR } 11.72 \\
(5.13,26.80)\end{array}$ & $<0.00001$ & $\begin{array}{c}\text { Moderate: } \\
\oplus \oplus \oplus 0\end{array}$ \\
\hline
\end{tabular}

Adverse No statistical analysis was performed. No adverse reactions (pain, compression, hematoma, burn) were observed effects in RCT

\begin{tabular}{|c|c|c|c|c|c|c|c|c|c|}
\hline \multirow[t]{2}{*}{$\begin{array}{l}\text { Liu et al. } \\
\text { (2018) }\end{array}$} & IIEF & Not serious & Serious & Not serious & Not serious & None & $\begin{array}{c}\text { MD 2.12 } \\
(0.62-3.62)\end{array}$ & 0.006 & $\begin{array}{c}\text { Moderate: } \\
\oplus \oplus \oplus \bigcirc\end{array}$ \\
\hline & EHS & Serious & Serious & Not serious & Not serious & None & $\begin{array}{c}\text { RD } 0.46 \\
(0.04-0.88)\end{array}$ & 0.03 & $\begin{array}{l}\text { Low: } \\
\oplus \oplus \bigcirc \bigcirc\end{array}$ \\
\hline
\end{tabular}

High: $\oplus \oplus \oplus \oplus$; Moderate: $\oplus \oplus \oplus$; Low: $\oplus \oplus \mathrm{O}$ O. LI-ESWT, low-intensity shockwave treatment; EHS, erection hardness score; ED, erectile dysfunction; PSV, peak systolic velocity; IIEF-EF, International Index of Erectile Function Erectile Function Domain score; RR, relative risk/ risk ratio; $\mathrm{MD}$, mean difference; $\mathrm{RD}$, risk difference; $\mathrm{OR}$, odds ratio; $\mathrm{WMD}$, weighted mean difference; SMD, standardized mean difference. 
showed that the IIEF-EF score of ED patients receiving more LI-ESWT treatment courses (10-12 times) was better than those with less treatment courses (5-6 times), and this difference was statistically significant, with weighted mean difference $(\mathrm{WMD})=1.81,95 \% \mathrm{CI}: 0.31,3.31, \mathrm{P}=0.02]$. Two SRs/MAs $(28,31)$ showed that LI-ESWT was effective for ED at 1 month, and 3, 6, and 12 months. Four SRs/MAs (29-31,33) scored ED patients, and one of them (30) showed that the efficacy of LI-ESWT for patients with mild ED was better than that for moderate and severe ED patients. In addition, two studies demonstrated that the curative effect of LI-ESWT was more superior for mild and severe ED than moderate ED (29,33), and one study (31) showed that LI-ESWT was effective for moderate to severe ED. Three SRs/MAs $(29,30,33)$ showed that more shock wave treatments could increase the IIEF-EF score $(\mathrm{MD}=2.86$; 95\% CI: $1.54,4.19, \mathrm{P}<0.0001 ; \mathrm{MD}=2.86,95 \% \mathrm{CI}: 1.54$, 4.19, $\mathrm{P}<0.01 ; \mathrm{MD}=5.11,95 \% \mathrm{CI}: 3.18,7.05, \mathrm{P}<0.00001$ ). Moreover, three studies $(29,30,33)$ analyzed energy flux density (EFD): Lu et al. (30) reported that EFD $>0.2 \mathrm{mj} / \mathrm{mm}^{2}$ could considerably improve the IIEF score ( $\mathrm{MD}=2.86,95 \%$ CI: $1.54,4.19, \mathrm{P}<0.0001)$, with no difference between LIESWT and sham treatment regarding EFD $<0.2 \mathrm{mj} / \mathrm{mm}^{2}$; Liu et al. (33) found that LI-ESWT (EFD $=0.09 \mathrm{~mJ} / \mathrm{mm}^{2}$ or EFD $>0.20 \mathrm{~mJ} / \mathrm{mm}^{2}$ ) was effective, with no difference between the two groups (EFD $=0.1-0.20 \mathrm{~mJ} / \mathrm{mm}^{2}$ ) ( $M D=0.00,95 \%$ CI: 2.23, 2.23, P=1.00). Man et al. (29) found that there were significant differences between $0.09 \mathrm{mj} / \mathrm{mm}^{2}$ and $0.14-0.25 \mathrm{mj} / \mathrm{mm}^{2}$ groups ( $\mathrm{MD}=4.14,95 \%$ CI: 0.87, 7.42, $\mathrm{P}=0.01) ; \mathrm{MD}=2.86,95 \%$ CI: $1.54,4.19, \mathrm{P}<0.0001)$.

Seven SRs/MAs (27-33) also compared the changes of EHS scores of LI-ESWT with sham treatment, and outcomes showed that LI-ESWT was better than sham treatment in the treatment of ED. One of seven SRs/ Mas (27) reported that LI-ESWT for ED patients with ineffective PDE5i was more effective than for ED patients with effective PDE5i, and the effect of continuous treatment for nine weeks was better than that for five weeks. Moreover, three SRs/MAs $(29,30,33)$ showed that LI-ESWT plus PDE5i and LI-ESWT monotherapy were effective in the treatment of ED, while the effect of combination therapy was more effective.

\section{Adverse events}

Among the eight included SRs/MAs, adverse events occurred in four $(27,28,31,32)$, and no adverse events occurred in the LI-ESWT group.

\section{Discussion}

\section{Summary of evidence}

Because the physiological mechanism of penile erection is complex, and there are many causes of ED. Clinically, the etiology of ED is divided into physiological, psychological and mixed, in which the mixed etiology accounts for the majority. Most of ED patients included in this paper evaluated ED with IIEF and EHS as outcome indicators. However, ED patients caused by psychological, neuroendocrine diseases, abnormal penis anatomy, radical prostatectomy, pelvic radiotherapy and penis transplantation will be excluded. This overview showed that many SRs regarding LI-ESWT for treating ED were published between 2017 and 2021, indicating a considerable increase in scientific interest in the use of LI-ESWT for ED treatment in past years. In comparison to sham therapy, LI-ESWT displayed solid curative effects in improving IIEF-EF, EHS, and PSV scores. The LI-ESWT group showed no obvious side-effects in the treatment of ED. The outcomes of this study supported LI-ESWT paratherapy for ED patients, but the evidence should still be treated with caution due to flaws in methodologies. Moreover, the number of shockwave treatments and energy flux density (EFD) affect the IIEF-EF, EHS and PSV scores.

\section{Implications}

The present overview is the first concerning SRs on the safety and efficacy of the use of LI-ESWT in ED treatment. In the eight included SRs, LI-ESWT was superior to sham treatment in improving ED symptoms. According to the safety assessment, LI-ESWT was generally safe and well-tolerated by patients during the treatment of ED. The evidence provided in the present study supported LI-ESWT for ED patients. Besides, LI-ESWT for ED patients with ineffective PDE5i was more effective than for ED patients with effective PDE5i. That is, LI-ESWT plus PDE5i and LI-ESWT monotherapy were both effective in the treatment of ED, while the combination therapy was more effective. However, we should be cautious about this evidence because of the methodological flaws of the included SRs.

Using AMSTAR-2, PRISMA, ROBIS, and other evaluation tools, we conducted a comprehensive analysis of the included SRs, and found that future research of this kind could be enhanced. Firstly, researchers should critically evaluate their work using AMSTAR-2, PRISMA, 
and ROBIS assessments to minimize the possibility of subjective bias and improve research quality. Secondly, the quality of the included literature is generally low, and it is essential to be registered in Prospero (http://www.crd.york. ac.uk/prospero) before the publication of a meta-analysis to lower the risk of bias. Thirdly, it is necessary to describe a comprehensive research strategy for a minimum of one database, and a list and explanation of excluded articles. Furthermore, in data synthesis analysis, if the heterogeneity of analysis results is significant, subgroup analysis or metaregression analysis is needed to avoid publication bias. Finally, the source of funding should be described, because the project sponsor may have influenced the research results.

Overall, LI-ESWT could improve the scores of IIEFEF, EHS, and PSV compared with sham treatment despite some flaws in the included eight SR/MAs. At the same time, the LI-ESWT for ED was generally safe and effective. In future, LI-ESWT for ED needs support through more high-quality articles.

\section{Limitations}

This overview has several limitations. Firstly, there was a lack of language diversity as only SRs published in Chinese and English were included, and as such findings do not reflect SRs published in other languages. The sample size of our study was relatively small. Moreover, it was also limited due to the potentially subjective process of quality evaluation. Individual subjective appraisals of the raters could have influenced their scoring, and as such results could vary. Thus, even though two independent raters were involved in the present overview the, results might have been affected by subjective ratings. Most of all, LIESWT in the treatment of ED has not been standardized, and there is still no unified standard for energy setting, treatment interval, type of ED patients, age of ED patients, treatment of combined diseases, disease duration of ED, etc. Therefore, in the process of clinical treatment, the whole condition of ED patients should be evaluated, and the individualized LI-ESWT scheme should be considered.

\section{Conclusions}

Our overview suggests that LI-ESWT may be beneficial for improving ED symptoms. However, due to certain limitations and inconsistencies, more rigorous, standardized, and comprehensive SRs are required to reach definitive conclusions to provide the basis for evidence-based clinical practice.

\section{Acknowledgments}

Funding: This study was supported by grants from the Key Research \& Development projects of the Science and Technology Department of Sichuan Province (2019YFS0184) and the 2019 "Apricot Scholars" Discipline Talent Research Promotion Plan (XSGG2019017).

\section{Footnote}

Reporting Checklist: The authors have completed the PRIMSA reporting checklist. Available at https://dx.doi. org/10.21037/tau-21-730

Conflicts of Interest: All authors have completed the ICMJE uniform disclosure form (available at https://dx.doi. org/10.21037/tau-21-730). The authors have no conflicts of interest to declare.

Ethical Statement: The authors are accountable for all aspects of the work in ensuring that questions related to the accuracy or integrity of any part of the work are appropriately investigated and resolved.

Open Access Statement: This is an Open Access article distributed in accordance with the Creative Commons Attribution-NonCommercial-NoDerivs 4.0 International License (CC BY-NC-ND 4.0), which permits the noncommercial replication and distribution of the article with the strict proviso that no changes or edits are made and the original work is properly cited (including links to both the formal publication through the relevant DOI and the license). See: https://creativecommons.org/licenses/by-nc-nd/4.0/.

\section{References}

1. Gratzke C, Angulo J, Chitaley K, et al. Anatomy, physiology, and pathophysiology of erectile dysfunction. J Sex Med 2010;7:445-75.

2. Campbell JD, Milenkovic U, Usta MF, et al. The good, bad, and the ugly of regenerative therapies for erectile dysfunction. Transl Androl Urol 2020;9:S252-61.

3. Fisher WA, Eardley I, McCabe M, et al. Erectile dysfunction (ED) is a shared sexual concern of couples I: couple conceptions of ED. J Sex Med 2009;6:2746-60. 
4. Gandaglia G, Briganti A, Jackson G, et al. A systematic review of the association between erectile dysfunction and cardiovascular disease. Eur Urol 2014;65:968-78.

5. Johannes CB, Araujo AB, Feldman HA, et al. Incidence of erectile dysfunction in men 40 to 69 years old: longitudinal results from the Massachusetts male aging study. J Urol 2000;163:460-3

6. Hall SA, Shackelton R, Rosen RC, et al. Risk factors for incident erectile dysfunction among community-dwelling men. J Sex Med 2010;7:712-22.

7. Jackson G, Montorsi P, Adams MA, et al. Cardiovascular aspects of sexual medicine. J Sex Med 2010;7:1608-26.

8. Seftel AD, de la Rosette J, Birt J, et al. Coexisting lower urinary tract symptoms and erectile dysfunction: a systematic review of epidemiological data. Int J Clin Pract 2013;67:32-45.

9. Kloner RA. Novel phosphodiesterase type 5 inhibitors: assessing hemodynamic effects and safety parameters. Clin Cardiol 2004;27:I20-5.

10. Mulhall JP, Jahoda AE, Cairney M, et al. The causes of patient dropout from penile self-injection therapy for impotence. J Urol 1999;162:1291-4.

11. Rompe JD, Kirkpatrick CJ, Küllmer K, et al. Doserelated effects of shock waves on rabbit tendo Achillis. A sonographic and histological study. J Bone Joint Surg Br 1998;80:546-52.

12. Gruenwald I, Appel B, Kitrey ND, et al. Shock wave treatment of erectile dysfunction. Ther Adv Urol 2013;5:95-9.

13. Vardi Y, Appel B, Jacob G, et al. Can low-intensity extracorporeal shockwave therapy improve erectile function? A 6-month follow-up pilot study in patients with organic erectile dysfunction. Eur Urol 2010;58:243-8.

14. Clavijo RI, Kohn TP, Kohn JR, et al. Effects of LowIntensity Extracorporeal Shockwave Therapy on Erectile Dysfunction: A Systematic Review and Meta-Analysis. J Sex Med 2017;14:27-35.

15. Fojecki GL, Tiessen S, Osther PJS. Effect of Linear LowIntensity Extracorporeal Shockwave Therapy for Erectile Dysfunction-12-Month Follow-Up of a Randomized, Double-Blinded, Sham-Controlled Study. Sex Med 2018;6:1-7.

16. Fukumoto Y, Ito A, Uwatoku T, et al. Extracorporeal cardiac shock wave therapy ameliorates myocardial ischemia in patients with severe coronary artery disease. Coron Artery Dis 2006;17:63-70.

17. Assaly R, Giuliano F, Clement P, et al. Extracorporeal Shock Waves Therapy Delivered by Aries Improves
Erectile Dysfunction in Spontaneously Hypertensive Rats Through Penile Tissue Remodeling and Neovascularization. Sex Med 2019;7:441-50.

18. Mattyasovszky SG, Langendorf EK, Ritz U, et al. Exposure to radial extracorporeal shock waves modulates viability and gene expression of human skeletal muscle cells: a controlled in vitro study. J Orthop Surg Res 2018;13:75.

19. Mulrow CD. Rationale for systematic reviews. BMJ 1994;309:597-9.

20. Shea BJ, Reeves BC, Wells G, et al. AMSTAR 2: a critical appraisal tool for systematic reviews that include randomised or non-randomised studies of healthcare interventions, or both. BMJ 2017;358:j4008.

21. Liberati A, Altman DG, Tetzlaff J, et al. The PRISMA statement for reporting systematic reviews and metaanalyses of studies that evaluate health care interventions: explanation and elaboration. PLoS Med 2009;6:e1000100.

22. Moher D, Liberati A, Tetzlaff J, et al. Preferred reporting items for systematic reviews and meta-analyses: the PRISMA statement. BMJ 2009;339:b2535.

23. Whiting P, Savović J, Higgins JP, et al. ROBIS: A new tool to assess risk of bias in systematic reviews was developed. J Clin Epidemiol 2016;69:225-34.

24. Guyatt GH, Oxman AD, Vist GE, et al. GRADE: an emerging consensus on rating quality of evidence and strength of recommendations. BMJ 2008;336:924-6.

25. Guyatt GH, Oxman AD, Kunz R, et al. GRADE guidelines: 2. Framing the question and deciding on important outcomes. J Clin Epidemiol 2011;64:395-400.

26. Balshem H, Helfand M, Schünemann HJ, et al. GRADE guidelines: 3. Rating the quality of evidence. J Clin Epidemiol 2011;64:401-6.

27. Zou ZJ, Tang LY, Liu ZH, et al. Short-term efficacy and safety of low-intensity extracorporeal shock wave therapy in erectile dysfunction: a systematic review and metaanalysis. Int Braz J Urol 2017;43:805-21.

28. Sokolakis I, Hatzichristodoulou G. Clinical studies on low intensity extracorporeal shockwave therapy for erectile dysfunction: a systematic review and metaanalysis of randomised controlled trials. Int J Impot Res 2019;31:177-94.

29. Man L, Li G. Low-intensity Extracorporeal Shock Wave Therapy for Erectile Dysfunction: A Systematic Review and Meta-analysis. Urology 2018;119:97-103.

30. Lu Z, Lin G, Reed-Maldonado A, et al. Low-intensity Extracorporeal Shock Wave Treatment Improves Erectile Function: A Systematic Review and Meta-analysis. Eur 
Urol 2017;71:223-33.

31. Dong L, Chang D, Zhang X, et al. Effect of Low-Intensity Extracorporeal Shock Wave on the Treatment of Erectile Dysfunction: A Systematic Review and Meta-Analysis. Am J Mens Health 2019;13:1557988319846749.

32. Mo DS, Zhan XX, Shi HW, et al. Efficacy and safety of low-intensity extracorporeal shock wave therapy in

Cite this article as: Yuan F, Wang Y, Ma Z, Jing M, You Y, Yu X, Chang D, Zhang P. Low-intensity extracorporeal shockwave therapy for erectile dysfunction: an overview of systematic reviews. Transl Androl Urol 2021;10(9):3684-3696. doi: 10.21037/tau-21-730 the treatment of ED: A meta-analysis of randomized controlled trials. Zhonghua Nan Ke Xue 2019;25:257-64.

33. Liu J, Jiang G, Liao B, et al. Erectile dysfunction: a metaanalysis of low-intensity extracorporeal shock wave therapy. Chongqing Medical Journal 2018;47:4033-8, 4043.

(English Language Editor: B. Meiser) 